\title{
DIAGNOSIS AND SURGICAL TREATMENT
}

\author{
By N. LLOYD RUSBY, D.M. (Oxon.), M.R.C.P. (Lond.) \\ (Physician to Out-patients, London Chest Hospital) \\ and \\ VERNON C. THOMPSON, F.R.C.S. (Eng.) \\ (Assistant Surgeon, London Chest Hospital)
}

The technical advances which have been made in the field of thoracic surgery in recent years are considerable, and have enabled the removal of one lung to be effected with only a small operative risk. Pneumonectomy offers to the victim of lung cancer his only reasonable hope of complete cure, a hope which, if it is to be fulfilled, depends as much upon the ability of the physician to recognise the disease at a sufficiently early stage as it does upon the skill and dexterity of the surgeon. An attempt is made in this article to outline the paths along which the diagnosis of carcinoma of the lung is first inferred and later made absolute; to recapitulate those points which seem to us valuable in assessing the suitability of a particular case for surgical treatment; and finally to give an account of the modern surgical technique employed. The differential diagnosis of intra-thoracic cancer is too large a subject to be incorporated.

Davidson (I930), in his book Cancer of the Lung, analysed ro7 cases of this disease observed at the Brompton Hospital and found that the six most prominent and frequent symptoms were cough, expectoration, dyspnoea, haemoptysis, pain in the chest, and loss of weight. This conclusion is substantially true to-day, and accordingly these symptoms will be submitted to closer examination.

Cough is almost invariably present. At first it is dry and irritating, and is productive only of a little mucoid secretion from the air-passages. Not uncommonly it is paroxysmal, and a sudden change of posture may excite a spasm. A cough of this character, appearing in middle life as an unusual phenomenon, which gradually increases in severity and is unassociated with an upper respiratory tract infection, should suggest the possibility of carcinoma.

Expectoration is customarily absent in the early stages, but appears subsequently and exhibits the greatest variation in both quantity and quality as the disease progresses, a variation that depends upon the character and position of the growth as well as the degree of accompanying bronchial obstruction. Every gradation is encountered from a trace of mucoid secretion voided only after forceful coughing to the easy elimination of frankly purulent, sometimes offensive, even bloodstained expectoration in an amount characteristically seen in bronchiectasis.

Dyspnoea is a symptom found with a frequency little less than cough and sputum, and in most examples of pulmonary cancer that are permitted to run their natural course difficulty in breathing, especially upon exertion, is complained of at some stage. Such dyspnoea may be caused only by exertion or after a severe fit of coughing, but it is intensified when the lumen of the bronchus becomes completely occluded and the corresponding volume of lung is rendered airless. Later, when the mediastinal glands round the lower end of the trachea and its bifurcation are heavily invaded respiratory difficulty may be urgent and orthopnoeic.

Pain was experienced by 50 per cent of the patients studied by Davidson (loc. cit.). Pain is generally referred to the chest, but it may be noticed in other situations such as the abdomen, shoulder, down the arm and upwards to the side of the neck. The mechanism of the production of pain in pulmonary cancer is not always easy to unravel in a particular case. It may be caused by the growth itself, by the pulmonary atelectasis to which it gives rise (although the gradual development of such a complication makes this cause an unusual one), or by spread to the mediastinal and cervical glands. The sensation of pain may be produced by extension of growth to the parietal pleura, by erosion of bone, by implication of posterior nerve roots as they emerge from the spine, and by interference with the brachial plexus. Thoracic pain of any kind, if it remains for any length of time unrelieved by simple remedies, deserves more detailed investigation. 
Haemoptysis is a specific complaint that should never be lightly dismissed, for the spitting of frank blood or staining of the sputum occurs commonly in malignant disease of the lung. In an adolescent or young adult haemoptysis should imply phthisis until this disease can be rigidly excluded: on similar reasoning the middle-aged patient with the same symptom should be as energetically investigated if carcinoma is to be recognised early.

Loss of weight is not an obvious manifestation of lung cancer in the early stages, and compared with some of the local symptoms is tardy in its development. A warning is needed lest absence of weight loss be taken as evidence against a diagnosis of malignant disease which seems reasonable on other grounds.

There are, in addition, a few less common manifestations, such as unexplained fever, loss of appetite, weakness, digestive disturbance, voice changes, and clubbing of the fingers which must be remembered and properly evaluated if unnecessary delay in diagnosis is to be avoided.

It is unfortunately true that no one symptom among those mentioned above is pathognomonic of early lung cancer, and all of them may be manifestations of other respiratory affections. The presence of malignant disease is seldom revealed, at a treatable stage, in a clear-cut and obvious manner, and is usually only inferred when two or more symptons are viewed in combination and related to the abnormal findings if any are found. In this way it is possible to recognise certain fairly well-defined modes of onset:

\section{Insidious}

The gradual evolution of symptoms in a subject of the " carcinoma " age (although younger age groups are by no means exempt) without any pre-existing respiratory disease is probably the commonest way for carcinoma of the bronchus to become manifest. The appearance of dry paroxysmal cough, which is later productive, associated with progressive dyspnoea on effort or chest pain should arouse a suspicion which becomes more firmly founded if haemoptysis, weakness or loss of weight supervene.

\section{Abrupt.}

(a) With sudden haemoptysis.

Such a mode of onset was found by Davidson (loc. cit.) in ro per cent of the ro7 cases that he studied. Rarely haemorrhage may be fatal before the cause can be ascertained, but otherwise bleeding occurring de novo or in an amount greater than streaking of the sputum in a bronchitic subject demands further action.

(b) Pneumonic form.

It is not uncommon to find carcinoma of the lung misdiagnosed at the beginning as pneumonia. Such a conclusion must be suspect if the illness runs an atypical course, is slow to resolve, or if repeated attacks of so-called "pneumonia" occur at short intervals. Moreover, if the free and satisfactory drainage of an empyema following pneumonia does not lead to the improvement expected the possibility of an underlying neoplasm should be entertained.

\section{Bronchitic.}

Under this heading are grouped those examples, lying midway between the insidious and abrupt modes of onset already described, which masquerade initially and sometimes for a dangerous length of time under the cloak of simple bronchitis. The distinguishing features of this variety are the failure of symptoms to respond to the customary treatment, the intensification of the illness under such treatment, and, occasionally, the detection of a " fixed " unilateral rhonchus on clinical examination.

\section{Pulmonary Suppuration.}

The clinical accompaniments of partial or complete bronchial obstruction due to neoplasm may be the presenting feature of the disease. Such accompaniments are pulmonary atelectasis, 
and suppuration which may be seen radiographically as a diffuse pneumonitis, a localised abscess cavity, or as an area of bronchiectasis. The possibility of a bronchial neoplasm being responsible for such changes should never be forgotten, and the view held by some workers that every case of " primary" lung abscess should be bronchoscoped once has much to recommend it.

\section{Dry. Pleurisy and Pleural Effusion.}

Of these two modes of onset pleurisy with effusion is the more common. Although tuberculosis as the cause of a pleural effusion in the middle-aged cannot be discounted, yet carcinoma should be uppermost in mind especially if exploratory puncture reveals a haemorrhagic fluid, and if the skiagram shows the mediastinum to be displaced towards the side of the lesion. Mediastinal shift in this direction suggests collapse or shrinkage of lung underneath the fluid, a finding which occurs in tuberculosis, but which is more commonly the result of neoplasm.

\section{Unusual Modes of Onset.}

(a) Appearance of Metastases.

Examples are occasionally seen in which the presenting symptoms and signs of the disease are referrable to enlargement of the lymphatic glands in the neck, especially in the supraclavicular fossae. The patient may complain either of the lump itself or of a pain in the shoulder radiating down the arm. Closely allied to this is that variety of the disease which starts in the extreme apex of the lung in the thoracic inlet, and was first described by Pancoast in I932 under the name "Superior pulmonary sulcus tumour."

Another important though rare manner in which attention may first be focussed upon a primary bronchial carcinoma is through the occurrence of cerebral secondaries, Elkington (I935), who has made a special study of metastases occurring in the brain, writes: ". . . there is no escape from the conclusion that bronchial carcinomata have a special tendency to give rise to secondary deposits in the brain." He found that in his series of 17 cases of secondary cerebral neoplasms the bronchus was the primary site of growth in 9 (52.9 per cent.). Koletsky (I938) approached the problem from the other direction, and analysed roo autopsies of carcinoma of bronchus. He found secondary deposits in the brain in 22 per cent.

(b) Abdominal symptoms.

That the earliest symptoms need not necessarily be referred to the thorax has been shown by Hochberg and Lederer (I939), who found in a series of 60 patients, I3 who fell into this group. In the majority of the 13 patients the most frequent complaints were epigastric distress, anorexia, nausea and vomiting.

(c) The only other unusual presenting symptoms that need be noted are change in the character of the voice due to recurrent laryngeal nerve palsy and, very occasionally, clubbing of the fingers.

\section{SPECIAL INVESTIGATIONS}

Consideration of the symptoms and modes of onset outlined above are sufficient by themselves only to excite suspicion of the presence of malignant disease. In order that the diagnosis may be completely established, special methods of investigation are necessary.

\section{Radiology.}

The skiagraphic appearances of carcinoma of the lung are fully dealt with by $\mathrm{Dr} . \mathrm{J}$. V. Sparks on another page. We do not intend, therefore, to trespass further upon this field except to emphasise that the X-ray film is the first and indispensable step in the elucidation of a doubtful case and to point out that the skiagraphic changes seen on the film are only rarely sufficiently distinctive to warrant a conclusive opinion as to the diagnosis. The use to which certain of the refinements of radiology can be put will be indicated in later sections.

\section{Bronchography.}

The use of radio-opaque oil to outline the bronchial tree in the diagnosis of this disease was more general a few years ago than it is now. By this means it is possible to demonstrate 
complete bronchial occlusion or a filling defect and also bronchial dilatation beyond the point of block. As bronchography, however, is almost always followed by bronchoscopy, a procedure which gives more exactly practically the same information, the employment of contrast radiography in this connection is of limited value.

\section{Bronchoscopy.}

In the majority of examples of carcinoma of the bronchus the growth can be clearly seen through a bronchoscope especially after pus or mucus have been aspirated, its position can be accurately localised, and a portion of it can be removed with biopsy forceps for histological study. In about 20 per cent of cases, however, the growth arises from a portion of the bronchial tree too distal to be seen through the bronchoscope (Tudor Edwards, (r938)). They may, nevertheless, supply indirect evidence of their presence, such as pressure upon the lumen of a bronchus from without, diminution in the normal respiratory movement of the bronchial tree, or a blunting of the usually sharp-edged carina due to enlarged glands at the bifurcation of the trachea. An endoscopic examination is incomplete unless both upper lobe orifices are inspected, if necessary with the aid of a retrograde telescope, and the whole length of the trachea and vocal cords are included. The information derived from bronchoscopy is of such inestimable value and the procedure can be conducted with such relative ease and safety under local anaesthesia that the indications for its use might profitably be widened.

\section{The Demonstration of Carcinoma Cells.}

It is often possible to obtain fragments of growth for histological examination by ways other than through the bronchoscope. The most important of these is the detection of tumour cells in the sputum by the special technique devised by Dudgeon and Patrick in I927 to whose paper and to those of Dudgeon and Wrigley (I935) and Barrett (I938) the reader is referred for details and results. (Barnard gives a brief outline of the method in his article-Ed.). Dudgeon claimed to be able to recognise in this way oat-celled, columnar-and squamous-celled types of neoplasm, a claim that has not passed entirely unrefuted, for other experienced pathologists, believing that the oat-celled tumour breaks down only as a terminal event, are rarely able to demonstrate cells of this type in the sputum (Gloyne, personal communication). This minor point of disagreement, however, does not detract from the intrinsic value of the method which, in practised hands, is great. A cautionary word is necessary against the examination of the sputum for neoplastic cells within ten days to a fortnight of a bronchoscopy as, following the trauma attaching to the procedure, cells suspicious of a squamous carcinoma may be shed and are occasionally seen in the sputum in the absence of any such growth. Other sources from which neoplastic tissue may be obtained are from the pleural fluid, from lung puncture, from biopsy of a lymphatic gland in neck or axilla, and occasionally from a nodule in the skin.

\section{Diagnostic Pneumothorax.}

For the diagnosis of certain types of tumour the induction of a pneumothorax is often of value. The solitary nodule, for instance, situated peripherally in the lung can be shown by this means to arise within the lung substance'and not from the chest wall.

In spite of the fact that the medical profession is becoming more alert in the diagnosis of carcinoma of the lung, it must be admitted that most patients, when diagnosed, have reached a stage of their disease past any possibility of radical treatment. This is not necessarily the fault of the patient or of the doctor, for it is often the extension of the growth beyond operable limits that causes the first symptom. The main hope of early diagnosis and of more successful treatment lies in the more frequent use of X-rays at routine examinations. Patients have been referred to us recently at an operable stage of malignant disease which had been discovered in the course of a barium meal investigation of the stomach, or by a routine skiagram taken for indefinite symptoms. These cases emphasise that an examination of the chest should always be made in the investigation of vague ill-health, and that such an examination is incomplete without an X-ray.

Some of the most favourable subjects from the operative point of view are those in whom the growth is not accessible to the bronchoscope, and in which there is no confirmation of the diagnosis by biopsy or by the discovery of carcinoma cells. The diagnosis then depends largely 
on the interpretation of an X-ray shadow. It may be said of these cases that when in the opinion of the surgeon the shadow represents an operable growth, and when this opinion is shared by his medical and radiological colleagues, the patient should be submitted to an exploratory thoracotomy. Once this opinion is formed there is no justification for further delay; by the time the diagnosis can be confirmed the growth may have become inoperable.

\section{THE DETERMINATION OF INOPERABILITY}

As pneumonectomy is the only radical treatment of carcinoma of the lung, a discussion of indications for the operation is less significant than what might be described as " the diagnosis of inoperability."

Exploratory thoracotomy is sometimes the only conclusive means of determining whether a carcinoma is operable or not. It is an operation that should be advised when it is thought that there is a favourable chance that the growth is capable of complete removal. Although the operation carries a low mortality and generally causes little post-operative discomfort, a fruitless inspection may cause harm, and even accelerate death. For this reason every possible step should be taken in the investigation of the patient to determine whether the growth has passed beyond the bounds of operability. In this connection special consideration should be given, in the general examination of the patient, to a search for distant metastases, the discovery of which obviously precludes operation. Persistent headache and minor changes in personality may denote cerebral metastases, while a degree of asthenia and hypotension inconsistent with the size and nature of the primary growth may indicate secondary deposits in the suprarenal glands. Careful palpation of the neck and axillae for enlarged lymph nodes, and of the liver for nodules or enlargement, is a first step in every instance.

\section{Invasion of Mediastinal Glands.}

A successful pneumonectomy is occasionally possible despite spread to this group of glands provided the degree of such involvement allows of a complete dissection of the affected glands. Heavier infiltration of the mediastinal glands, sufficient to preclude surgery, is often obvious on the ordinary skiagram; indeed in some examples (the lympho-sarcomata of a generation ago) it may be impossible at operation or autopsy to decide where growth ends and the mediastinal secondary mass begins. In an advanced stage of the process the obstructive " mediastinal syndrome" can be detected with such clinical manifestations as engorgement of the veins in the neck and over the chest, swelling of the face and arms, cyanosis and urgent dyspnoea Information with regard to less extensive involvement can be derived from a widening of the upper mediastinal shadow on an X-ray film, particularly a displacement of the superior vena cava to the right, while a tomographic study of the lung root will sometimes demonstrate a glandular mass which was not obvious on the ordinary film.

\section{Involvement of Nerves.}

Huskiness of the voice, progressing to aphonia, may be the result of a recurrent laryngeal nerve palsy, and confirmation of this can be sought from an examination of the vocal cords, while a high diaphragm with paradoxical movement as seen on the screen is almost certainly the result of interference with the function of the phrenic nerve. In only a few isolated cases with the latter complication is pneumonectomy possible.

It is not uncommon for a growth to extend sufficiently far back to involve the posterior nerve roots as they emerge from the spinal column. The complaint of constant pain over an area supplied by one or more segments with some corresponding disturbance of sensation should be viewed with suspicion. Extension upwards to the brachial plexus has already been mentioned in connection with Pancoast's tumour, while Horner's syndrome, due to encroachment upon the sympathetic chain, is another characteristic feature of apical tumours of this type.

\section{Erosion of Bone.}

Erosion of the vertebral column, ribs or sternum by growth is one cause for the pain of lung cancer, and is a complication that contra-indicates operation, unless the involvement is confined to one or two ribs that can be removed en masse with the lung. Bony changes can 
often be seen in the ordinary chest film; but they are made more obvious by special methods of X-ray technique.

\section{Invasion of the Pericardium and Heart Muscle.}

This can be suspected from the appearance of two signs which have been observed by us on rare occasions. The first is the detection of pericardial friction. Although extension to the pericardium does not necessarily place a patient beyond the hope of surgery, clearly, in such a circumstance, an operation of this magnitude cannot be enthusiastically recommended.

The second of these two signs is the sudden appearance of auricular fibrillation, often paroxysmal, in a patient in whom other well-established causes of this type of abnormal rhythm are absent. We have recently observed a man aged 37 years with a proved carcinoma of bronchus which gave every indication of being operable. He complained of attacks of " palpitations" which were shown electrocardiographically to be due to auricular fibrillation. At operation the growth was found to have invaded the heart muscle in the neighbourhood of the left auricle.

\section{Pleural Effusion.}

As a general rule pleural effusion suggests that the tumour is inoperable; whether this is so or not depends upon the mechanism of its production, which may be one or more of the following:-

(a) Lymphatic obstruction, due to mediastinal deposits.

(b) Involvement of the pleura by growth, in which case the effusion is often haemorrhagic.

(c) Inflammatory reaction when the growth is accompanied by secondary infection.

(d) Massive collapse of the lung, when the effusion may be transitory.

Whereas in the first two instances the growth is inoperable, in $(c)$ and $(d)$ the presence of fluid does not in itself contra-indicate operation.

\section{Bronchoscopic Appearances.}

There are certain definite points of evidence to be derived from bronchoscopic examination, such as paralysis of a vocal cord, gross distortion and narrowing of the trachea or main bronchi, widening and fixation of the carina, or extension of the growth to within half an inch of the carina. All these render radical removal of the lung impossible.

\section{The Patient.}

In assessing the prospect of successful surgery, attention should not be riveted exclusively upon the local lesion: of equal importance is the general condition of the patient. Can he withstand one of the most severe operations in modern surgery?

The presence of cardio-vascular disease must be carefully sought and evaluated. Other systemic disorders such as kidney disease, diabetes and alcoholism should engender caution and will influence judgment. The functional efficiency of the sound lung, which will subsequently have to perform the work of two, must be investigated: whereas younger patients can easily and quickly adjust themselves to the altered physiology after pneumonectomy, the same is not true of the elderly. In those over the age of 60 the cardio-respiratory reserve is possibly already at a low level, and bronchitic and emphysematous changes are commonly found. No precise figure of cardio-respiratory reserve has ever been worked out but careful attention directed to the blood pressure, response to exercise, and to vital capacity estimations before and after artificial pneumothorax has been induced, will assist in selecting suitable subjects.

Another important consideration is the degree of pleural adhesion, for if this is extensive the time taken at operation may be prolonged and the amount of blood lost increased to dangerous limits. A patient who would survive the removal of a non-adherent left lung might succumb to the removal of an adherent right lung; for it must be remembered that the left lung comprises only 45 per cent of the total lung volume.

The final judgment, however, must be left to the experience of the surgeon who knows the technical difficulties likely to be encountered in any individual case. 


\section{Choice of Operation.}

\section{SURGICAL TREATMENT}

Malignant growths of the lung are usually so situated that for technical reasons alone it is necessary to remove the whole lung in order to remove the growth.

When the anatomy of the lung is considered, with its intercommunication of lymphatics as they approach the hilum, it is clear that anything less than total pneumonectomy will fail to remove the main area of lymphatic drainage from an individual lobe. Moreover, it becomes obvious that in order to perform a radical operation, the bronchus must be divided as near the carina as possible, and the lymph nodes must be removed together with the lung. Such an operation can only be carried out by dissection and individual division of the structures of the hilum.

The operation of pulmonary lobectomy carries a lower mortality and is less disabling than total pneumonectomy, and can, therefore, be carried out successfully in some patients who would not survive pneumonectomy. For this reason it has, in the writers' opinion, a small but definite place in the treatment of carcinoma of the lung. While not the operation of choice, lobectomy gives some hope, when technically possible, to those patients who would not survive the more radical operation; but it should not be employed unless total pneumonectomy is contra-indicated.

\section{Operative Complications.}

Although it is usual to discuss the complications of an operation after its description, it seems more fitting to the writers that these should be discussed first. It is a surgeon's duty even before the preparation of the patient, to plan every step so that complications do not occur; only by considering these beforehand can he hope for success in his object.

In chest surgery the maxims of general surgery hold good, but certain considerations of anatomy and physiology make them sometimes impossible to fulfil. It is one of the principles of surgery that dead spaces should be obliterated to diminish the chances of haematoma formation and infection. Owing to the rigidity of the chest wall it is often impossible to do this, and after pneumonectomy the whole hemithorax remains as a dead space. Careful haemostasis and a rigid aseptic technique, therefore, become of paramount importance.

\section{Complications Occurring During the Operation.}

I. Shock and haemorrhage.

2. Obstruction of the opposite bronchial tree by "spill-over" of blood or secretions from the affected lung.

3. Anoxia due to mediastinal displacement.

4. Sudden arrest or irregularity of the heart beat due to reflex vagal effects.

\section{Post-operative Complications.}

I. Anoxia.

2. Shock.

3. Affections of the opposite lung, such as oedema, bronchitis, pneumonia and atelectasis:

4. Broncho-pleural fistula. This may, if small, give rise to a pressure pneumothorax. If large, it may cause sudden flooding of the opposite lung with pleural exudate. In any event it leads to infection of the pleural space.

5. Empyema.

6. Secondary haemorrhage.

The above complications may be expected to occur after pneumonectomy unless careful precautions are taken to prevent them. Other post-operative sequelae which may occasionally occur and which are less under the control of the surgeon are acute dilatation of the stomach, cerebral abscess, and pulmonary embolism.

\section{Preparation of the Patient.}

It is advisable to allow the patient a moderate amount of exercise before operation, in order to maintain the general tone of the muscles as well as that of the heart muscle.

A pneumothorax should be induced two or three weeks before the operation. Not only does this aid in assessing the operability of the lesion and the technical difficulties that may 
arise, but it causes a shunting of a part of the pulmonary circulation to the sound lung and gives time for a certain amount of physiological adjustment to take place.

During this period anaemia should be treated by blood transfusion, and the patient should be given an adequate supply of vitamin $\mathrm{C}$.

If there is any sputum, the patient should be given postural drainage three times a day. This type of drainage is particularly important on the day of operation.

Breathing exercises of the Brompton Hospital type should be given during this period of preparation, so that the patient will make the best use possible of the sound lung.

\section{Anaesthetic.}

The services of an anaesthetist skilled and experienced in chest work are invaluable. It is the advances in anaesthesia and radiology that have made modern chest surgery possible.

Although there are many individual variations in technique, the usual preference is for a general anaesthetic using nitrous oxide, oxygen and cyclopropane, which allows a high oxygen concentration to be maintained. A comfortable induction with intravenous evipan or pentothal is usually employed, and trilene can be substituted for cyclopropane in the early stages of the operation, while diathermy is being used for coagulating blood vessels in the chest wall.

Some method of maintaining positive pressure is necessary so that anoxia due to mediastinal displacement may be dealt with when it occurs. For this purpose a close fitting face piece is usually adequate, but some anaesthetists prefer more complicated methods.

It is also important that the anaesthetist should maintain a good air-way by an endotracheal tube, through which or around which another tube may be passed to aspirate secretions from the trachea or main bronchi.

In order to prevent "spill-over" into the sound lung the writers prefer to block the main bronchus on the affected side. For this purpose, before the induction of the anaesthetic, the bronchoscope is passed and a special hydrostatic bag is introduced and distended to obstruct the bronchus. A tube passes through the bag and may be brought through the anaesthetic mask, thus enabling air and secretions to escape direct from the affected lung without soiling the trachea or the opposite bronchial tree. By the use of this appliance patients with as much as one pint of sputum a day have been successfully operated upon without any anaesthetic difficulties.

\section{THE TECHNIQUE OF PNEUMONECTOMY}

In order to minimise shock it is wise to start a drip blood transfusion at the beginning of the operation. Care should be taken to give the blood slowly and not to give a quantity greater than the amount of fluid lost. Owing to the additional strain thrown on the pulmonary circulation by pneumonectomy oedema of the lung is likely to occur unless the transfusion is carefully regulated.

\section{Approach.}

There are two main methods of approach, the anterior and the postero-lateral.

For the anterior approach the patient lies in the dorsal position; a transverse incision is made along the 3 rd intercostal space and is carried down through the pectoral and intercostal muscles and pleura, the 3 rd and 4 th intercostal cartilages are divided and the wound is opened with a mechanical rib spreader. Another anterior incision which is employed is a curved incision running along the $5^{\text {th }}$ space below the breast and then upwards over the costal cartilages. The $5^{\text {th }}, 4^{\text {th }}$, and $3^{\text {rd }}$ costal cartilages may be divided and a flap of chest wall can be displaced upwards.

For the postero-lateral approach the patient lies in the lateral position with the upper arm hanging over the edge of the table. An incision is made along the line of the 6th rib from a point $2^{\prime \prime}$ from the spine to the mid-axilla. The muscles are divided along the line of the incision and the 6 th rib is resected. The pleural cavity is opened through the rib bed. A wide exposure is then obtained by spreading the ribs with a mechanical retractor.

The postero-lateral approach is the one in most common use, it undoubtedly gives an excellent exposure and allows access to the whole of the pleural cavity.

The anterior approach gives direct access to the hilum of the lung, but it may involve the operator in considerable difficulty when the lung is adherent to the chest wall. 
When the chest has been opened the first step is carefully to palpate the tumour and to define the exact limits of its direct extension. If the growth is infiltrating the pericardium or the great vessels, the operation should be abandoned and the chest closed. The presence of a large fixed mass of glands in the mediastinum also renders further interference useless, but if the glands can be removed together with the lung the operation of pneumonectomy should be continued.

\section{Freeing the Adherent Lung.}

In most instances some degree of pleural adhesion between the visceral and parietal pleura is present. If the adhesion is the result of recent inflammation the pleural surfaces may be gently separated with the fingers. Adhesions of old standing often have to be cut with scissors and bleeding vessels ligatured. In some cases the pleural symphysis is so close that the layers cannot be separated, it is then necessary to strip the parietal pleura from the chest wall by working in the extra-pleural plane.

\section{Ligation of the Pulmonary Vessels.}

When all adhesions have been separated and the lung is quite free, the pulmonary ligament is divided between artery forceps and ligatured. The pleura around the hilum is then cut with scissors and the dissection and individual ligation of the pulmonary vessels may be commenced. These vessels consist of the pulmonary artery and the superior and inferior pulmonary veins. They should be divided between ligatures in that order. It is advisable to use linen thread or silk, and to apply two proximal ligatures, tieing the second in the groove made by the first. When dividing the vessel, a good flange should be left distal to the ligature to avoid the possibility of the vessel retracting. It is at this stage of the operation that technical difficulties are likely to arise when the growth is near the hilum. There may not be room to apply distal ligatures, and recourse must be made to artery forceps applied to the vessel as it enters the lung.

On the right side the pulmonary artery is so short that it may have to be ligatured distal to its two primary branches.

\section{Closure of the Bronchus.}

When the vessels have been divided, the lung is left attached only by the bronchus. At this stage a special bronchial clamp is applied as near the carina as possible. The clamp is so constructed that the soft posterior wall of the bronchus is made to approximate the cartilaginous anterior wall, and the lumen is closed without crushing. As the clamp is closed, the anaesthetist deflates and withdraws the intra-bronchial balloon that was introduced at the beginning of the operation. The bronchus is then divided one centimetre distal to the clamp, the lung is removed, and the bronchial mucous membrane is immediately treated with pure carbolic acid. This procedure maintains asepsis and serves to destroy the mucous membrane.

Closure of the bronchus presents the greatest technical problem of pneumonectomy. No method of closure yet devised is so reliable that the surgeon can feel confident that a bronchial fistula will not subsequently develop. Many complicated methods of closure have been employed, but in the writers' experience the simple method has been most successful. A row of mattress sutures of fine linen thread is applied immediately distal to the clamp, and the edges of the bronchus are then closed with interrupted sutures of the same material. Finally the clamp is removed, and the mediastinal pleura is approximated over the bronchial stump.

\section{Closure of the Chest.}

Before closing the chest a careful inspection should be made and all bleeding should be checked, particular attention being paid to the bronchial artery which may have escaped the bronchial sutures.

As a precaution against infection, sulphapyridine powder may be scattered over the cavity.

The ribs are approximated and held in position by three pericostal sutures of strong catgut. The intercostal muscles are sutured with continuous catgut, and the two muscle layers and the skin are closed without drainage.

While the patient is still on the table in the lateral position it is advisable to remove some air from the chest, and to adjust the pressure in the hemithorax so that it is slightly negative. 
When the patient is turned on to his back, the pressure will rise, as the effect of gravity in displacing the mediastinum to the opposite side is removed.

\section{Post-operative Care.}

In the immediate post-operative period the usual steps must be taken to combat shock, and the patient should be given oxygen by the B.L.B. mask.

As soon as possible he should be raised to the semi-sitting position and the intra-thoracic pressure on the operated side should be adjusted so that it is atmospheric.

During the first six weeks after operation it is advisable to aspirate fluid from the chest as required and to maintain atmospheric pressure in the hemithorax. If a bronchial fistula occurs, or if the fluid becomes infected, the chest should be drained by rib resection. When a fistula occurs drainage may be a very urgent matter to prevent an overflow into the remaining lung. In these cases immediate intercostal drainage should be performed in the ward. Such emergencies are heralded by the expectoration of pleural fluid and may be avoided by maintaining a dry pleural cavity by aspiration.

After six weeks it is unlikely that a bronchial fistula will develop, and aspirations may be discontinued.

As the air in the hemithorax is absorbed the cavity tends to fill with exudate in which fibrinous clot is deposited, so that ultimately a multilocular semi-fluid mass fills the space. At the same time the hemithorax greatly diminishes in size as a result of elevation of the diaphragm, displacement of the mediastinum, and approximation of the ribs.

Thus by a combination of these mechanisms the space that was once the size of the hemithorax becomes obliterated.

Finally, it is evident that the treatment of cancer of the lung by surgery demands the combination of specialised technique and co-ordinated team-work between all departments of the hospital or unit, both before and after operation. This is almost as necessary a prerequisite for success as early diagnosis, and in this respect carcinoma of the lung is no exception to a rule which applies to malignant disease occurring in other vital organs of the body.

\section{REFERENCES}

BARRETT, N. R. (1938), J. Thorac. Surg., 8, r69.

DAVIDSON, M. (1930), Cancer of the Lung, John Wright, Bristol.

DUDGEON, L. S., and PATRICK, C. V. (I927), Brit. J. Surg., 15, 250.

DUDGEON, L. S., and WRIGLEY, C. H. (1935), J. Laryngol. and Otol., 50, 752.

ELKINGTON, J. St. C. (1935), Proc. R. Soc. Met., 28, 1080.

EDWARDS, A. TUDOR (1938), Brit. J. Surg., 26, 166.

HOCHBERG, L. A., and LEDERER, M. (1939), Arch. Int. Med., 63, 80.

KOLETSKY, S. (1939), Arch. Int. Med., 62, 636.

\section{SPECIAL NUMBERS OF THE JOURNAL}

Special numbers have been published from time to time, each dealing comprehensively with a particular branch of medicine or surgery. These special numbers are as follows, and copies, price 2s. each, post free, may be obtained from the Fellowship of Medicine, I Wimpole Street, London, W.I. A list of contents of any individual number will be sent on application.

\begin{tabular}{|c|c|c|}
\hline OBSTETRICS & (August, r933) & Tropical Diseases \\
\hline OPHTHALMOLOGY & (August, 1934) & Cerebral Tumours \\
\hline NEurology & (April, 1935) & (February, 1940) \\
\hline UROLOGY & (November, 1935) & *WaR WOUNDS OF THE ChEST \\
\hline CARDIOLOGY & (April, 1936) & *WAR WOUNDS OF THE ABDOMEN \\
\hline Proctology & (August, 1936) & *WAR WOUNDS OF THE LIMBS \\
\hline NEPHRITIS & (February, 1937) & *WAR Wounds OF THE EYE AND ORBIT (June, r940) \\
\hline RHEUMATISM & (September, r937) & *WaR Wounds of the Head and Face (July, r940) \\
\hline ORTHOPEDICS & (October, r937) & \\
\hline
\end{tabular}

